\title{
THE CLINICAL PERFORMANCE OF ALL CERAMIC ZIRCONIA-VENEERED CROWNS AFTER DIFFERENT SURFACE TREATMENTS OF ZIRCONIA FRAMEWORK: 30 MONTHS CLINICAL ASSESSMENT
}

\author{
Haitham Amr* and Mazen A. Attia**
}

\begin{abstract}
Objective: To evaluate the clinical performance of zirconia-veneered crowns after different surface treatment of zirconia frameworks named as; no surface treatment, sandblasting, silicatization (CoJet ${ }^{\mathrm{TM}}$ sand), Selective infiltration etching (SIE) and the novel acids mixture.
\end{abstract}

Materials and methods: A total of 50 patients of age ranged from 20 to 35 years were presented with root canal-treated molar teeth that needed post-retained restorative treatment. They were divided into 5 groups according to surface treatment of zirconia frameworks (10cases each); Group S1: Zirconia frameworks received no surface treatment. Group S2: Zirconia frameworks received sandblasting with $110 \mu \mathrm{m}$ aluminum oxide airborne particle abrasives.GroupS3: Zirconia frameworks received CoJet sand surface treatment. Group S4: Zirconia frameworks received selective infiltration etching surface treatment. Group S5: Zirconia frameworks received a specially formulated mixture of hydrofluoric acid (HF $9 \mathrm{wt} \%$ ) and nitric acid (HNO3 $26 \mathrm{wt} \%$ ) at a ratio of (4:1) for $5 \mathrm{~min}$. All Zirconia frameworks received IPS e.max Ceram veneering porcelain. They received all ceramic zirconia based single crowns using Cerec in-Lab CAD/CAM technology for designing and milling zirconia frameworks. Teeth preparation was performed to achieve $2 \mathrm{~mm}$ occlusal clearance, $1.5 \mathrm{~mm}$ axial reduction and $1 \mathrm{~mm}$ rounded shoulder subgingival finish line. Secondary impression was taken for each case using addition silicone rubber base impression material and poured using special reflective cerec stone (CAM Base) to facilitate scanning of the preparation using the in-Lab extra oral scanner "inEos". 3D virtual models were obtained that were used for designing zirconia frameworks. Then, milling of zirconia blocks were performed using inLab Cerec 3 milling machine to obtain zirconia frameworks followed by sintering procedures for the zirconia frameworks. After try in of sintered zirconia frameworks in the patient's mouth, they received different types of surface treatment. After that, porcelain build up was conducted by layering technique. All ceramic zirconia based crowns were then cemented using dual-cured resin cement (Multilink Auto Mix) and were subjected to a period of 6, 12, 18, 24 and 30 months clinical observation according to the modified USPHS criteria for indirect restorations, where prosthodontic results were recorded. Statistical analysis was performed using a commercially available software program (SPSS 19; SPSS, Chicago, IL, USA). Values were presented as number and percentages. Chi square test was used to compare categorical data.The level of significance was set at $\mathrm{P}<0.05$.

* Lecturer of fixed prosthodontics, Faculty of Dentistry, Fayoum University

** Lecturer of Fixed Prosthodontics, Faculty of Dentistry, Beni Suef University 
Results: It was found that at 6 months, score A was recorded in all specimens of IPS e.max Ceram, with no statistically significant difference between different surface treatments $(p=1)$. At 12 months, score A was recorded in all specimens, with no statistically significant difference between different surface treatments $(\mathrm{p}=1)$. At 18 months, score A was recorded in $100 \%$ specimens of CoJet and acid mixture surface treatment, in $90 \%$ of specimens of no treatment and in $80 \%$ of specimens of sandblasting and SIE; with no statistically significant difference between different surface treatments $(\mathrm{p}=0.815)$. At 24 months, score A was recorded in $80 \%$ specimens of sandblasting, CoJet and acid mixture surface treatment, in $66.7 \%$ of specimens of no treatment and in $22.2 \%$ of SIE; with a statistically significant difference between different surface treatments $(p=0.045)$. At 30 months, score A was recorded in $80 \%$ specimens of sandblasting, $88.9 \%$ CoJet and $77.8 \%$ of acid mixture surface treatment, in $44.5 \%$ of specimens of no treatment and in $11.1 \%$ of SIE; with a statistically significant difference between different surface treatments $(\mathrm{p}=0.0024)$.

Conclusions: (1) In all cases, the tested systems showed chipping of the comparable lowstrength veneering ceramic but no fracture of the high-strength core or the natural tooth in clinical cases. (2) The treatment of the surface zirconia framework with Cojet system was able to maintain acceptable clinical performance at 24 months and 30 months recall periods. However, the other surface treatments showed lesser performance. (3)No direct correlation between zirconia framework surface treatments and long term bonding to veneering ceramic could be addressed; however, it could be multifactorial such as lack of proper framework support, internal defects, direction, magnitude and frequency of the applied load as well as the residual stresses induced by processing.

KEY WORDS: 30 months clinical assessment, all ceramic zirconia-veneered crowns, surface treatments of zirconia, bonding of veneering porcelain to zirconia.

\section{INTRODUCTION}

All-ceramic crowns and fixed partial dental structures have become increasingly popular over the past decade. Ceramic materials are being used as single layer restorations for many decades. One of the greatest disadvantages of these single layer ceramic restorations is their relative low strength. Attempts to reinforce the glassy matrix of these materials resulted in opaque ceramics that lack the required translucency, opalescence, and fluorescence which originally gave these ceramics their attractive esthetic characteristics ${ }^{(1)}$.

The introduction of high strength Yttria partially stabilized tetragonal zirconia frameworks (Y-TZP) opened up the design and application limits of all-ceramic restorations with more success and reliability. Nowadays, zirconia is used as core and a framework structure for the construction of all ceramic crowns and fixed partial dentures which are further layered with esthetic veneering ceramics to obtain the required esthetics. These bi-layered restorations combine both strength and esthetics and thus can be used both in the anterior and the posterior region of the mouth ${ }^{(2)}$. Combining the strength of ceramic cores and superior esthetics of a weaker veneer ceramic can result in a reliable and more biocompatible restoration that make long span and complex all-ceramic restorations possible due to the unique and excellent mechanical properties of zirconia. With CAD/CAM technology, the design and production of zirconia frameworks became a completely digitalized process $^{(3)}$.

Many factors must be considered during fabrication of bi-layered all-ceramic restoration such as the design of the framework, thickness of the veneering ceramic, proper selection of the luting agent, insertion technique, and the expected functional loads. Other important factors are related to the properties of the selected materials such 
as difference in thermal expansion coefficients (DCTE), surface finish of the framework material, layering technique of the veneering ceramic, firing program, and cooling stresses ${ }^{(4)}$.

Chipping of the veneering ceramic seems to be the major problem with zirconia frameworks. Complication rates of $15 \%$ after two years have been reported $^{(5)}$. In a prospective clinical trial chipping was observed in $13 \%$ of all reconstructions after three years ${ }^{(6)}$ and $15 \%$ of all reconstructions after 5 years $^{(7)}$. These failure rates are significantly higher compared to metal-ceramic restorations ${ }^{(8)}$. Different factors may cause inferior core-veneer bond strength. Well known factors are pre-stresses due to differences in thermal expansion coefficients (CTE) of the core and veneer ceramics, poor wetting of the core by the veneer ceramic, firing shrinkage of veneering ceramic, transformation of zirconia crystals at core-veneer interface due to thermal influences or stress loading and inherent flaws formation during various fabrication steps ${ }^{(9,10)}$.

A variety of surface preparation techniques have been advocated to improve the bonding of the veneering ceramic such as; the use of acids, hot etching solution, silica coating, airborne particle abrasion, liners and selective infiltration etching. Several attempts have investigated the effect of application of acids as a surface treatment for zirconia ceramic with contrasting results. Previous studies used hydrofluoric acid with various concentrations and etching times, as well as application of hot etching solution which increased the surface roughness of zirconia and created microspaces that would optimize the overall bonding mechanism..$^{(11-14)}$ A novel acid mixture treatment has been tried in previous studies and was claimed to improve the bond strength between the core and the veneering porcelain. It consists of a specially formulated mixture of hydrofluoric acid (HF 9 $\mathrm{wt} \%$ ) and nitric acid (HNO3 $26 \mathrm{wt} \%$ ) at a ratio of $(4: 1){ }^{(13,14)}$
Therefore, it was found beneficial to evaluate the clinical performance of zirconia-veneered crown after different surface treatment of zirconia framework named as; no surface treatment, Sandblasting, silicatization $\left(\right.$ CoJet $^{\mathrm{TM}}$ sand), Selective infiltration etching (SIE) and the novel acids mixture.

\section{MATERIALS AND METHODS}

\section{Patients selection:}

A total of 50 male patients participated in this clinical study. They received all ceramic zirconia based single crowns with the veneering surface of their frameworks receiving different types of surface treatment tested: no surface treatment, Sandblasting, silicatization $\left(\mathrm{CoJet}^{\mathrm{TM}}\right.$ sand) and Selective infiltration etching (SIE) and the novel acids mixture that consists of a specially formulated mixture of hydrofluoric acid (HF $9 \mathrm{wt} \%$ ) and nitric acid (HNO3 $26 \mathrm{wt} \%$ ). The patients were subjected to 30 months observation period where clinical results were recorded. The age of the patients ranged from 20 to 35 years. They were presented with root canal-treated molar teeth that needed post-retained restorative treatment. Clinical and radiographic examinations of the cases were performed and the data were recorded and analyzed to determine patients that fulfilled the requirements of the study.

\section{Criteria of patients' selection}

Oral hygiene: the patients selected had good oral hygiene conditions, low caries index, low plaque and bleeding indices and maintained good oral hygiene measures.

Endodontic condition: the selected teeth were examined radiographically for the root canal treatment performed and checked for the periapical condition and quality of the root canal filling. Any suspected cases were retreated first or disregarded completely.

Periodontal condition: normal periodontal status was mandatory for the selected teeth showing 
normal gingival conditions (color, inflammation, bleeding...), normal mobility levels (grade 1), normal probing depth, and normal alveolar bone level shown proximally on the radiographs.

Any cases that did not meet the above mentioned criteria were disregarded in an attempt to standardize the study. All the selected teeth received $0.8 \mathrm{~mm}$ titanium post (unimetric)* and composite core (MulticoreFlow) ${ }^{* *}$ and received all ceramic zirconia based crowns as final restorations. As regard to the opposing occluding teeth, $75 \%$ were unrestored natural teeth and $25 \%$ had restorations.

\section{Clinical grouping}

The 50 patients were divided according to the type of preveneering surface treatment of zirconia frameworks into 5 groups (10cases each):

- Group $\mathbf{S}_{\mathbf{1}}$ : Zirconia frameworks received no surface treatment.

- Group $\mathbf{S}_{2}$ : Zirconia frameworks received sandblasting with $110 \mu \mathrm{m}$ aluminum oxide airborne particle abrasives.

- GroupS : Zirconia frameworks received CoJet sand surface treatment.

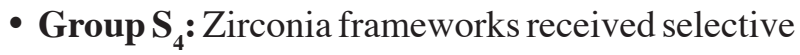
infiltration etching surface treatment.

- Group $\mathbf{S}_{5}$ : Zirconia frameworks received a specially formulated mixture of hydrofluoric acid (HF 9 wt\%) and nitric acid (HNO3 26 $\mathrm{wt} \%)$.

All Zirconia frameworks received IPS e.max Ceram veneering porcelain.

The materials used in this study, their descriptions, compositional structures and manufacturer are presented in table (1).

\footnotetext{
* Dentsply,USA

** Ivoclar- vivadent, Schaan, Leichstenstein, Switzerland *** Sirona, Bensheim, Germany
}

\section{Clinical and technical procedures}

The clinical and technical procedures are demonstrated in figure 1. After full mouth scaling and polishing, preoperative radiographs, digital photographs and primary impressions to obtain study model were taken for every patient. Tooth preparation was done using advanced preparation set for cerec restoration (Intensive, USA). Tooth preparation was done to achieve $2 \mathrm{~mm}$ occlusal clearance, $1.5 \mathrm{~mm}$ axial reduction and $1 \mathrm{~mm}$ rounded shoulder subgingival finish line (figure 1). Secondary impression was taken for each case with putty wash impression technique using addition silicon rubber base impression material (Panasil,Kettenbach GmbH \& CO.KG, Germanay) after tissue retraction using retraction cord inserted in place by aid of retraction cord applicator (Roeko, Coltene Whaledent, Switzerland). The impression was poured using special reflective cerec stone (CAM Base, Sirona, Germany) to facilitate scanning of the preparation. Another impression was taken to obtain a hard stone model to be used in surface treatment procedures so not to destroy the original cast. Moreover, a sectional rubber base impression was taken to construct provisional restoration that was cemented using temporary cement (Urbical, Promedica, Germany).

\section{Cerec in-Lab CAD/CAM frameworks fabrica- tion}

\section{Step 1: Scanning of the die}

The restoration data were entered and the tooth number was then highlighted on the diagram and framework was selected for the restoration box. The in-Lab extra oral scanner "inEos" "*** was used for this purpose. A top view scan was performed to obtaine 3D virtual model. 
TABLE (1) The materials used in this study

\begin{tabular}{|c|c|c|c|c|}
\hline $\begin{array}{l}\text { Product } \\
\text { Name }\end{array}$ & Description & Compositional structure & $\begin{array}{l}\text { B a t c h } \\
\text { Number }\end{array}$ & Manufacturer \\
\hline $\begin{array}{l}\text { IPS e.max } \\
\text { ZirCAD } \\
\text { (C15L) }\end{array}$ & $\begin{array}{l}\text { Yttrium partially } \\
\text { stabilized tetragonal } \\
\text { zirconium dioxide } \\
\text { blocks(Y-TZP) }\end{array}$ & $\begin{array}{l}\mathrm{ZrO}_{2} 87-95 \% \text { wt. and additional contents: } \mathrm{HFO}_{2} \text {, } \\
\mathrm{Al}_{2} \mathrm{O}_{3}, \mathrm{Y}_{2} \mathrm{O}_{3} \text { and other oxides. }\end{array}$ & M24219 & \multirow{2}{*}{$\begin{array}{l}\text { Ivoclar- } \\
\text { vivadent, } \\
\text { Schaan, } \\
\text { Leichstenstein, } \\
\text { Switzerland }\end{array}$} \\
\hline $\begin{array}{l}\text { IPS e.max } \\
\text { Ceram }\end{array}$ & $\begin{array}{l}\text { Veneering porcelain } \\
\text { system }\end{array}$ & $\begin{array}{l}\text { Low-fusing nano-fluorapatite glass-ceramic, } \\
\text { Components: } \mathrm{SiO}_{2}>60 \% \text { wt. Additional contents: } \\
\mathrm{Al}_{2} \mathrm{O}_{3}, \mathrm{ZnO}_{2}, \mathrm{Na}_{2} \mathrm{O}, \mathrm{K}_{2} \mathrm{O}, \mathrm{ZrO}_{2}, \mathrm{CaO}, \mathrm{P}_{2} \mathrm{O}_{5} \text {, fluoride, } \\
\text { and pigments }\end{array}$ & L09526 & \\
\hline $\begin{array}{l}\text { COBRA } 110 \\
\mu \mathrm{m}, \text { White }\end{array}$ & $\begin{array}{l}\text { Airborne particle } \\
\text { abrasion }\end{array}$ & $110 \mu \mathrm{m}$ aluminium oxide particles & $\mathrm{J} 32015$ & $\begin{array}{l}\text { Renfert, } \\
\text { Germany }\end{array}$ \\
\hline CoJet $^{\mathrm{TM}}$ sand & $\begin{array}{l}\text { silica coated } \\
\text { abrasive particles }\end{array}$ & $30 \mu \mathrm{m}$ Silica coated aluminium oxide particles & D402776 & $\begin{array}{l}\text { 3M ESPE AG, } \\
\text { Germany }\end{array}$ \\
\hline $\begin{array}{l}\text { Selective } \\
\text { infiltration } \\
\text { etching agent }\end{array}$ & $\begin{array}{l}\text { low melting point } \\
\text { infiltration glass } \\
\text { powder }\end{array}$ & $\begin{array}{l}\text { silicon }(\mathrm{Si}) 30 \mathrm{wt} \% \text {, titanium }(\mathrm{Ti}) 13 \mathrm{wt} \% \text {, aluminum } \\
(\mathrm{Al}) 8 \mathrm{wt} \% \text {, potassium }(\mathrm{K}) 3 \mathrm{wt} \% \text {, rubidium }(\mathrm{Rb}) 1 \\
\text { wt } \% \text {, and magnesium }(\mathrm{Mg}) 1 \mathrm{wt} \% \text {, and the rest was } \\
\text { oxygen }\left(\mathrm{O}_{2}\right) \text {. }\end{array}$ & & Custom made \\
\hline $\begin{array}{l}\text { Novel acid } \\
\text { mixture }\end{array}$ & $\begin{array}{l}\text { Specially } \\
\text { formulated mixture } \\
\text { of } \mathrm{HF} \text { and } \mathrm{HNO} 3\end{array}$ & $\begin{array}{l}\text { hydrofluoric acid (HF } 9 \mathrm{wt} \%) \text { and nitric acid (HNO3 } \\
26 \mathrm{wt} \%) \text { at a ratio }(4: 1)\end{array}$ & & Custom made \\
\hline $\begin{array}{l}\text { Multicore } \\
\text { flow }\end{array}$ & $\begin{array}{l}\text { Injectable } \\
\text { composite }\end{array}$ & $\begin{array}{l}\text { Base and Catalyst paste: } 28 \mathrm{wt} \% \text { Dimethacrylates, } \\
54 \mathrm{wt} \% \text { Barium glass fillers, Ba-Al-fluorosilicate } \\
\text { glass, highly dispersed silicon dioxide, } 16 \mathrm{wt} \% \\
\text { Ytterbium trifluoride, } 1 \mathrm{wt} \% \text { catalysts, stabilizers and } \\
\text { pigments }\end{array}$ & J26668 & \multirow[t]{2}{*}{$\begin{array}{l}\text { Ivoclar- } \\
\text { vivadent, } \\
\text { Schaan, } \\
\text { Leichstenstein, } \\
\text { Switzerland }\end{array}$} \\
\hline $\begin{array}{l}\text { Multilink } \\
\text { Automix }\end{array}$ & $\begin{array}{l}\text { Adhesive resin } \\
\text { cement }\end{array}$ & $\begin{array}{l}\text { Baseand CatalystPastes:22-26wt\%dimethacrylates,6- } \\
7 \mathrm{wt} \% \text { hydroxyethyl methacrylate (HEMA), Barium } \\
\text { glass filler and Silica filler Ytterbiumtrifluoride, } \\
\text { initiators, stabilizers and pigments }\end{array}$ & J09235 & \\
\hline
\end{tabular}

\section{Step 2: designing the framework on the software}

The 3D virtual model displayed on the design window was then used to design the framework with the help of the software given tools in the view and design windows. The preparation was trimmed to hide unnecessary regions on the model. The preparation margin "finish line" was entered. The insertion axis was positioned vertically to the occlusal surface of the model. Then, the software built up the framework according to the previous given parameters for the e.max ZirCAD framework which involve the thickness of the framework (0.6mm circular and $0.7 \mathrm{~mm}$ occlusal) and $(40 \mu \mathrm{m})$ provided cement space. Finally, all surfaces of the framework were checked for any design error before milling.

\section{Step 3: Milling process}

In the milling preview, the type and size of the block were selected as" e.max ZirCAD" and size $(\mathrm{C} 15 \mathrm{~L})$ then the milling process was started. 


\section{Step 4: Sintering process}

The framework was trimmed carefully using diamond abrasives at very low speed to remove excess material at the site of connection with the ceramic block and then, the sintering procedure for the zirconia frameworks was conducted according to the instructions in the relevant equipment of the supplier as previously mentioned for the zirconia specimens. Then, dies preparation was performed and the sintered frameworks were seated each over its corresponding die and checked for presence of any marginal defects. Try in of the sintered zirconia framework in the patient mouth was carried out to check the marginal adaptation, occlusal and proximal contact of the framework.

\section{Surface treatment of zirconia frameworks}

\section{Sandblasting}

Every surface of the zirconia framework was airborne particle abraded with $110 \mu \mathrm{m}$ aluminum oxide particles $\left(\mathrm{AL}_{2} \mathrm{O}_{3}\right)$ at $2.8 \mathrm{MPa}$ pressure using sandblaster device and all surfaces of the frameworks were faced to the sand beam for $15 \mathrm{sec}$ followed by ultrasonic cleaning in distilled water for 10 minutes.

\section{Cojet treatment}

Every surface of the zirconia framework was treated with $30 \mu \mathrm{m}$ Cojet Sand at $2.8 \mathrm{MPa}$ pressure using air prophy unit (Lily 101-M4) mounted on the dental unit. All surfaces of the frameworks were faced to the nozzle opening for $15 \mathrm{sec}$.

\section{Selective infiltration etching}

The veneering surfaces of the zirconia frameworks were received selective infiltration etching surface treatment. The surfaces of zirconia discs were coated with a low melting point infiltration glass using compressed air. A syringe connected to an air compressor was filled with the infiltration glass and the compressed air was used to cover the surfaces of zirconia frameworks with it Then, zirco- nia frameworks were heated to $750{ }^{\circ} \mathrm{C}$ (at a rate of $60{ }^{\circ} \mathrm{C} / \mathrm{min}$ ) using a computer programmed electrical induction oven (VULCAN 3-550, DEGUSSA-NEY DENTAL, USA) and was held for $2 \mathrm{~min}$ and then cooled to room temperature. Zirconia frameworks were selectively etched to dissolve the infiltration agent by immersion in 5\% hydrofluoric acid bath in ultrasonic cleaner for $15 \mathrm{~min}$. Finally all frameworks were cleaned in water bath and air dried

\section{Novel acid mixtures:}

A specially formulated mixture of hydrofluoric acid (HF $9 \mathrm{wt} \%$ ) and nitric acid (HNO3 $26 \mathrm{wt} \%$ ) was prepared in the National Institute of Research, Chemistry of surface treatment Department, in a ratio 4:1 Between hydrofluoric and nitric acid. Solution was placed in plastic container and zirconia frameworks were immersed in the solution at room temperature for 5 minutes. The frameworks were washed with distilled water then left to dry in a heat oven at $100^{\circ} \mathrm{C}$ for 1 hour.

\section{Porcelain build up}

After try in of sintered zirconia frameworks in the patient's mouth and performing the required surface treatment for their veneering surfaces, porcelain build up was conducted. The veneering ceramic for the zirconia frameworks were fabricated by one experienced dental technician according to the manufacturer's instructions. Porcelain build up was performed using IPS e.max Ceram veneering porcelain with its corresponding liner ZirLiner as recommended by the manufacturer. The veneering porcelain was fired in a porcelain oven according to its suggested firing schedule. The layering procedures were conducted according to the manufacturer instructions using dentin and incisal materials. Dentin and Incisal firing was performed following the stipulated firing parameters. Finishing diamonds were then used to achieve a natural shape and surface structure of the restoration, such as growth lines and convex/concave areas. Stain firing was conducted with Shade material. 


\section{Cementation procedures}

Dual-cured resin cement Multilink Auto Mix (Ivoclar- vivadent, Schaan, Leichstenstein, Switzerland) was used for cementation of zirconia based restorations. The temporary restoration was firstly removed from the patient mouth together with residues of the temporary cement from over the preparation. The preparation was rinsed with water spray. Subsequently, was dried with water and oil-free air. Necessary corrections were performed using a special cerec set (Intensive,USA) of fine diamonds for intraoral correction at high speed with copious water spray and with slight pressure. Ground surfaces were polished and isolation of the operative area was conducted with cotton rolls and saliva ejector.

To optimize the bond strength to Zirconium Oxide frameworks, chemical adhesion with the placement of the Metal/Zirconia primer was performed. A thin layer of the Metal/Zirconia primer was applied with a micro-brush and was let to react for $3 \mathrm{~min}$. Subsequently, was dried with water and oil-free air. The two Multilink Primer liquids A and B were mixed in a 1:1 mixing ratio (1 drop of Primer A and 1 drop of Primer B). The mixed Multilink Primer A/B was applied with a micro-brush on the entire preparation surface starting from the finish line and scrubbing with slight pressure for $15 \mathrm{sec}$ and a reaction time of $15 \mathrm{sec}$ was permitted. The applied primer was subsequently dried. As the primer is solely self-curing, no light-curing was necessary. Multilink Automix was directly applied to the inner surface of the restoration with the desired quantity taking care to be applied only on the axial surfaces not to interfere with the seating of the restoration on the corresponding preparation. The restoration was seated and fixed in place and the cement was cured briefly with light (1-2 sec), excess material was removed. Subsequently, all margins were light cured for 20 seconds.

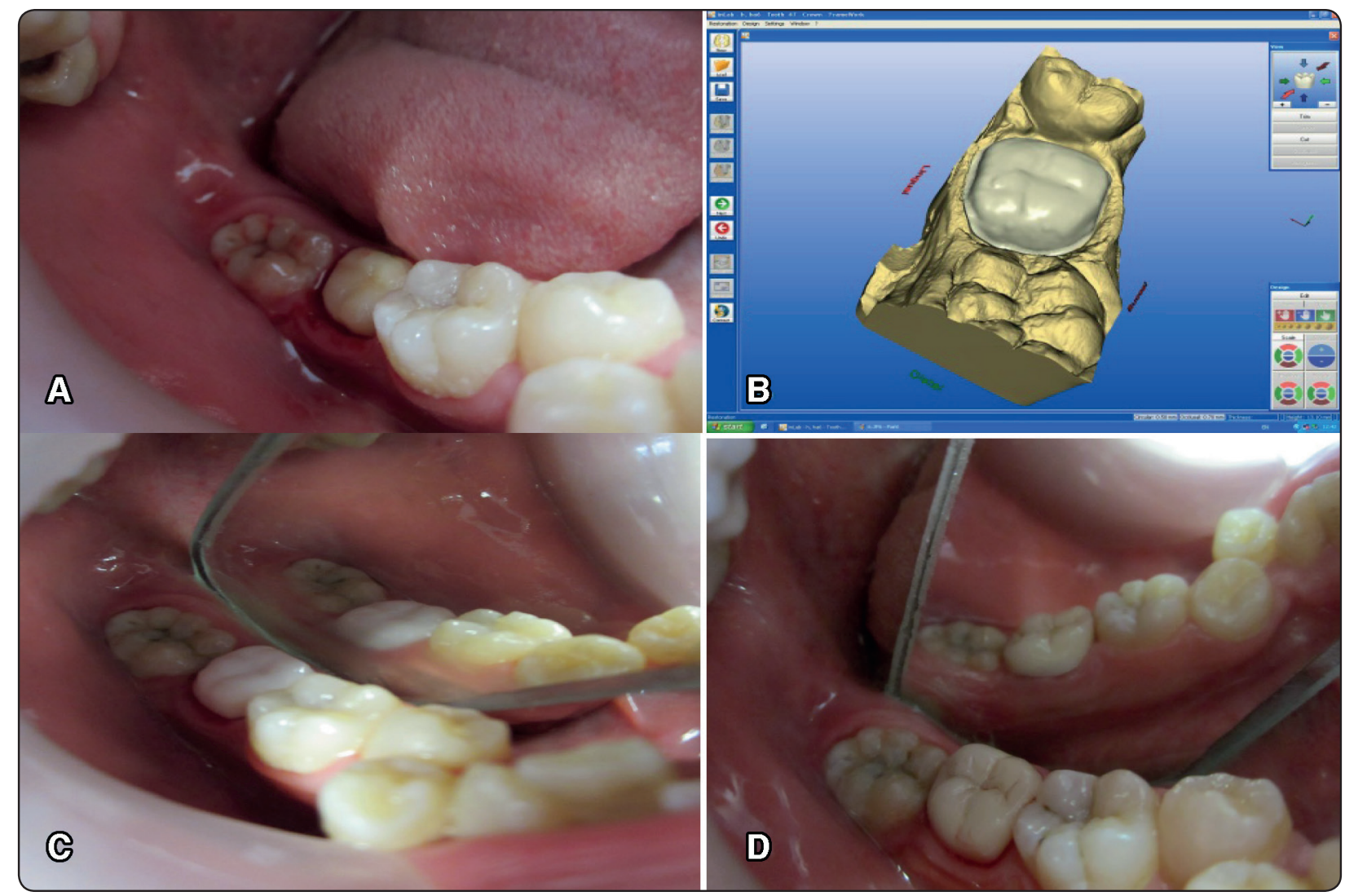

Fig. (1) Clinical steps of zirconia veneered crown A: tooth preparation, B: designing zirconia framework on software, C: Try in of the sintered zirconia framework in the patient mouth, D: cemented zirconia veneered crown 


\section{Follow up and detection of failure}

Patients recall was performed after 6 months, 12 months, 18 months, 24 months and 30 months for clinical evaluation according to the clinical evaluation criteria (modified USPHS criteria for indirect restorations). A 4-point score was used to designate the status for each assessed category. The scoring system was as follows: Alpha: excellent result, restorations without changes or clinically ideal; Bravo: acceptable result, restorations with changes that are clinically acceptable and do not require replacement; Charlie: unacceptable, restorations with major changes that require replacement to prevent further deterioration; Delta: unacceptable, immediate replacement necessary.

Clinical evaluation of restoration in terms of fracture resistance using modified Ryge criteria ${ }^{(13)}$

Alpha A: Smooth surface (shiny after air drying)

Bravo B: Dull surface and/or chipping of porcelain that does not impair function

Charlie C: Chipping of the veneering porcelain impairing esthetics and function and/or exposing framework material.

Delta D: Fracture of the connector between the pontic and the retainer and/or fracture through the framework material

\section{Statistical analysis}

Statistical analysis was performed using a commercially available software program (SPSS 19; SPSS, Chicago, IL, USA). Values were presented as number and percentages. Chi square test was used to compare categorical data.

The level of significance was set at $\mathrm{P}<0.05$.

\section{RESULTS}

Comparison between different surface treatments within the same observation time

Comparison between different surface treatments within the same observation time is presented in table 2, Fig. 2. At 6 months, score A was recorded
TABLE (2) Comparison of The clinical performance of zirconia-veneered crown after different surface treatment (percentage of A, B, C scores), (Chi square)

\begin{tabular}{|c|c|c|c|c|}
\hline \multirow{2}{*}{ Time } & \multirow{2}{*}{ Surface treatment } & \multicolumn{3}{|c|}{ Failure Score } \\
\hline & & $\mathrm{A}$ & B & $\mathrm{C}$ \\
\hline \multirow{6}{*}{$6 \mathrm{M}$} & No treatment & $100 \%$ & 0 & 0 \\
\hline & Sandblasting & $100 \%$ & 0 & 0 \\
\hline & CoJet & $100 \%$ & 0 & 0 \\
\hline & SIE & $100 \%$ & 0 & 0 \\
\hline & Acids mixture & $100 \%$ & 0 & 0 \\
\hline & $\mathrm{P}$ & \multicolumn{3}{|c|}{$1 \mathrm{~ns}$} \\
\hline \multirow{6}{*}{$12 \mathrm{M}$} & No treatment & $100 \%$ & 0 & 0 \\
\hline & Sandblasting & $100 \%$ & 0 & 0 \\
\hline & CoJet & $100 \%$ & 0 & 0 \\
\hline & SIE & $100 \%$ & 0 & 0 \\
\hline & Acids mixture & $100 \%$ & 0 & 0 \\
\hline & $\mathrm{P}$ & \multicolumn{3}{|c|}{$1 \mathrm{~ns}$} \\
\hline \multirow{6}{*}{$18 \mathrm{M}$} & No treatment & 90 & 10 & 0 \\
\hline & Sandblasting & 80 & 20 & 0 \\
\hline & CoJet & $100 \%$ & 0 & 0 \\
\hline & SIE & 80 & 20 & 0 \\
\hline & Acids mixture & $100 \%$ & 0 & 0 \\
\hline & $\mathrm{P}$ & \multicolumn{3}{|c|}{$0.815 \mathrm{~ns}$} \\
\hline \multirow{6}{*}{$24 \mathrm{M}$} & No treatment & 66.7 & 33.3 & 0 \\
\hline & Sandblasting & 80 & 20 & 0 \\
\hline & CoJet & 88.9 & 11.1 & 0 \\
\hline & SIE & 22.2 & 66.7 & 11.1 \\
\hline & Acids mixture & 88.9 & 11.1 & 0 \\
\hline & $\mathrm{P}$ & \multicolumn{3}{|c|}{$0.045^{*}$} \\
\hline \multirow{6}{*}{$30 \mathrm{M}$} & No treatment & 44.4 & 33.3 & 22.2 \\
\hline & Sandblasting & 80 & 20 & 0 \\
\hline & CoJet & 88.9 & 11.1 & 0 \\
\hline & SIE & 11.1 & 44.45 & 44.45 \\
\hline & Acids mixture & 77.8 & 22.2 & 0 \\
\hline & $\mathrm{P}$ & \multicolumn{3}{|c|}{$0.0024 *$} \\
\hline
\end{tabular}

Significance levelp $<0.05,{ }^{*}$ significant,$n s=n o n-$ significant 
in all specimens, with no statistically significant difference between different surface treatments $(\mathrm{p}=1)$. At 12 months, score A was recorded in all specimens with no statistically significant difference between different surface treatments $(\mathrm{p}=1)$. At 18 months, score A was recorded in $100 \%$ specimens of CoJet and acid mixture surface treatment, in $90 \%$ of specimens of no treatment and in $80 \%$ of specimens of sandblasting and SIE; with no statistically significant difference between different surface treatments $(\mathrm{p}=0.815)$. At 24 months, score
A was recorded in $80 \%$ specimens of sandblasting, CoJet and acid mixture surface treatment, in $66.7 \%$ of specimens of no treatment and in $22.2 \%$ of SIE; with a statistically significant difference between different surface treatments $(\mathrm{p}=0.045)$. At 30 months, score A was recorded in $80 \%$ specimens of sandblasting, $88.9 \%$ CoJet and $77.8 \%$ of acid mixture surface treatment, in $44.5 \%$ of specimens of no treatment and in $11.1 \%$ of SIE; with a statistically significant difference between different surface treatments $(\mathrm{p}=0.0024)$.

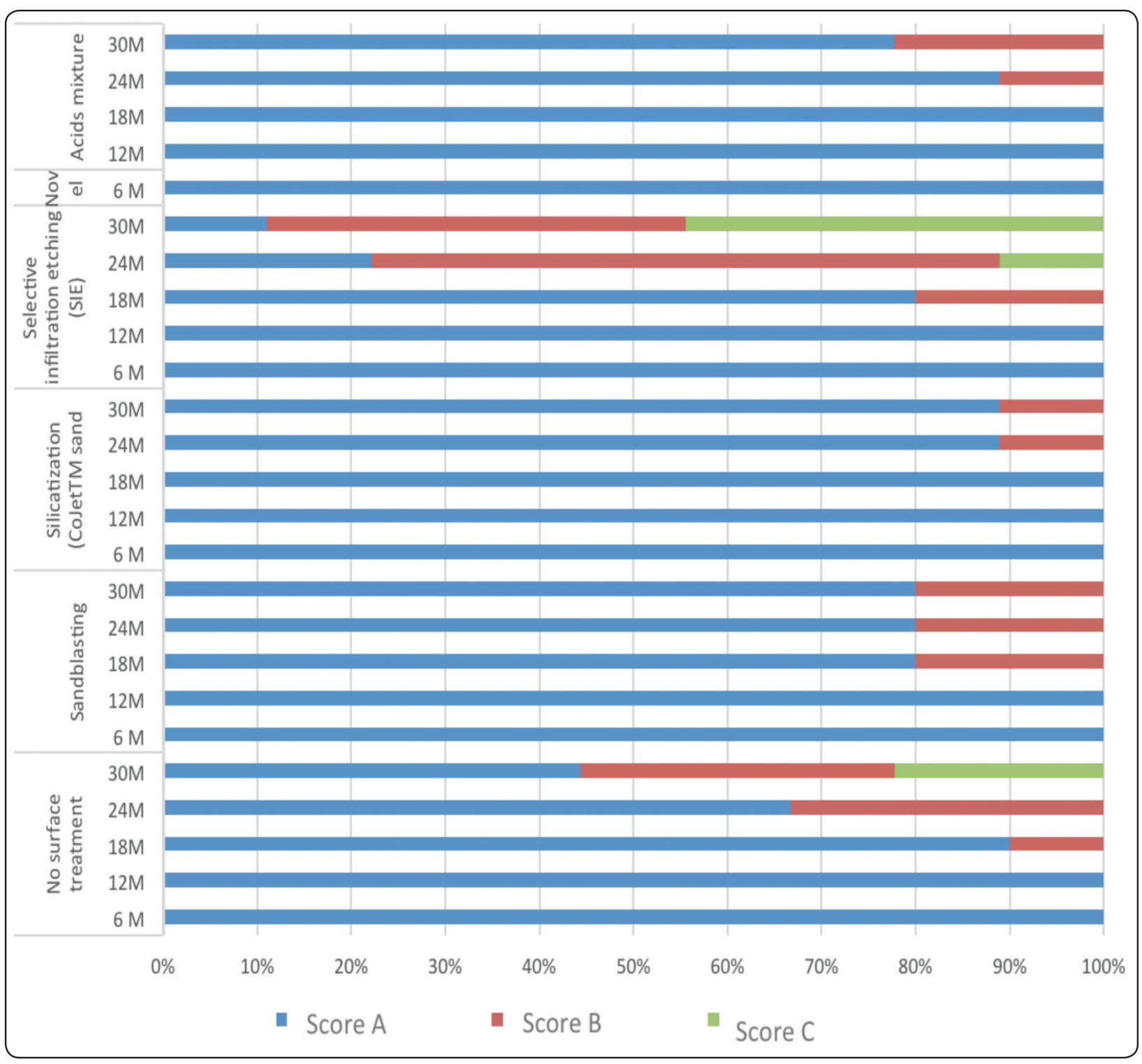

Fig. (2) Bar chart showing the clinical performance of zirconia-veneered crown after different surface treatment 

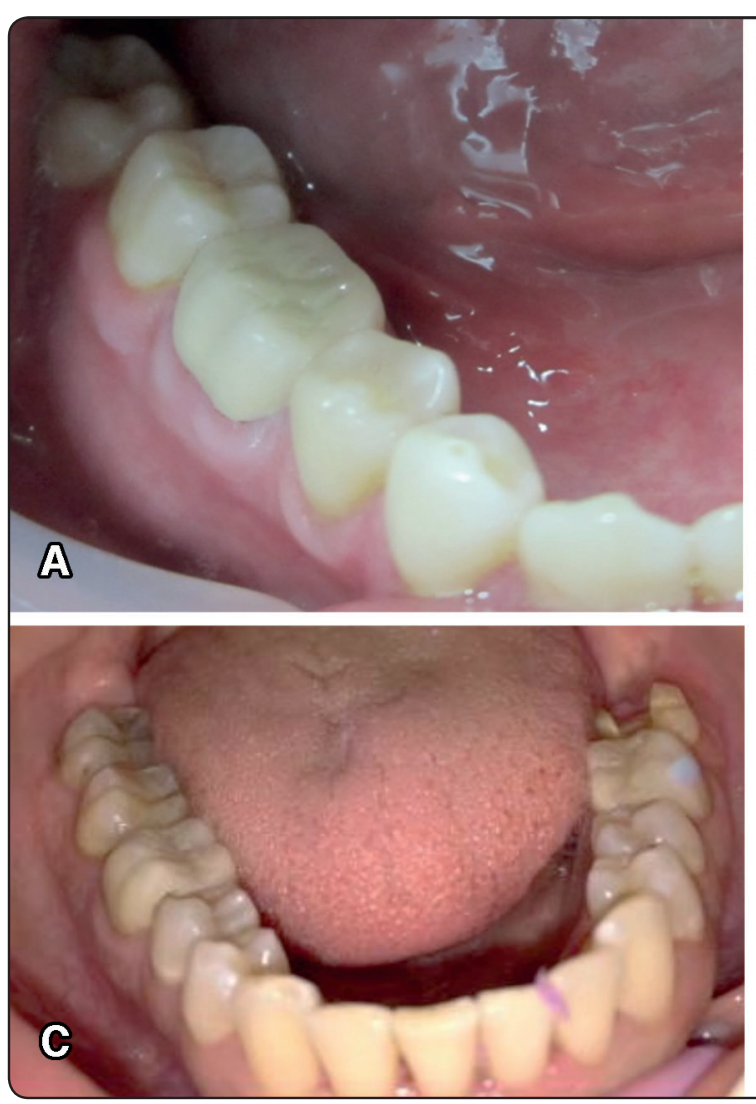

\section{DISCUSSION}

All-ceramic crowns had become increasingly popular over the past decade. Zirconia based allceramic restorations are possible substitutes for the strong but less esthetic ceramo-metalic restorations in which a layer of veneering ceramic is fired on the surface of zirconia framework to provide the restoration with the required color and esthetics. This combination has very attractive aesthetic and biocompatibility properties in addition to its high strength and fracture toughness. However, high chipping rates of veneering porcelain for zirconia based restorations have been reported in clinical studies as stated by Aboushelib et al 2010 ${ }^{(11)}$.

Using different types of surface treatment for zirconia veneering surface to improve bond strength with overlaying veneering porcelain is still a questionable matter. Therefore the present study was designed to evaluate and compare the effect of

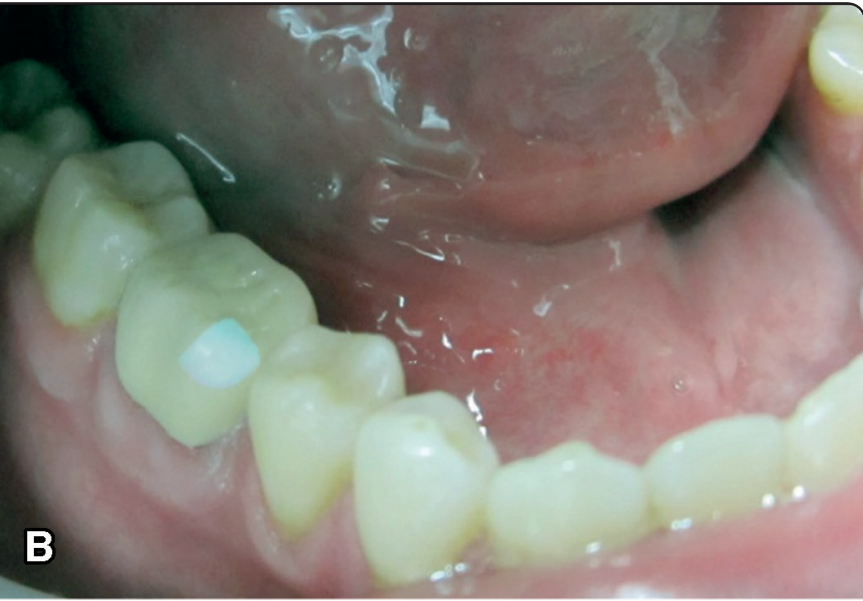

Fig. (3) the clinical performance of zirconia-veneered crown after different surface treatment after different recall period: A) after 6 months, B) after 24 monts showing veneering ceramic "pop-off" at functional cusp in SIE group, C) after 30 months recall with veneering ceramic "pop-off" at functional cusp at the left side in no surface treatment group and an acceptable clinical performance at the right side in Cojet group.

different types of zirconia surface treatments named as; Sandblasting, silicatization (CoJet ${ }^{\mathrm{TM}}$ system), selective infiltration etching (SIE) and novel acids mixture on the clinical performance of zirconiaveneered crown as framework/veneer bonding is susceptible to chemical, thermal, and mechanical influences under intraoral conditions. Moreover, some studies have proposed that the application of thermal cycling induces repeated stress on the core/ veneer interface, resulting in a weakened bond as stated by Saito et al 2010 ${ }^{(10)}$. Regarding the novel acid mixture, on combining the two acids; hydrofluoric acid and nitric acid, the etching solution resulted in modifications to the zirconia surface morphology. This idea is adopted from the technique used for pickling of stainless steel alloys. The action of this acid solution is basically a corrosion-controlled process. It can be speculated that the acid solution may determine a chemical dissolution of the grain structure on the zirconia surface, enlarging the grain 
boundaries throughout the preferential removal of the less-arranged, high-energy peripheral atoms. Etching rate depends on solution movement over the ceramic surface, which in turn depends on both mixing ratios and immersion time. The presence of nitric acid in the novel acid mixture used in the current study seems to enhance the etching effect of hydrofluoric acid on zirconia ceramic.

Y-TZP blocks were selected for this study as zirconia properties are largely dependent on both the starting powder and fabrication technique. The zirconium oxide powder must be as pure as possible and the particle size sufficiently small to allow optimal mechanical properties. An accurate concentration and uniform distribution of yttrium oxide is essential for stabilizing of zirconia crystals, thus promoting the more stable tetragonal phase formation. The optimal sintering process should be performed between $1400{ }^{\circ} \mathrm{C}$ and $1500{ }^{\circ} \mathrm{C}$ to allow the material to reach satisfactory mechanical characteristics as stated by Casucci et al 2010 ${ }^{(16)}$.

The concept behind selection e.max Ceram veneering systems was the slight CTE mismatch between e.max and Y-TZP blocks and this was supposed to generate compressive stresses in the weaker veneering porcelain, thereby enhancing the overall strength of the restorations as stated by Saito et al $2010^{(10)}$.

The concept behind using different types of surface treatment was to evaluate the effect of alternative chemo-mechanical treatments on the surface microstructure of zirconia core for zirconia based restorations since variations in surface roughness and topography affect the bond strength at zirconia -veneer interface as mentioned by Casucci et al 2010 ${ }^{(16)}$.

As the average maximum sustainable biting force is approximately 170 pounds and it varies markedly from one area of the mouth to another and from one individual to another, so for standardization purpose, the participated patients in this clinical study were selected to be males of age ranged from 20 to 35 years. They all were presented with root canal-treated molar teeth. Advanced preparation set for cerec restoration was used to provide almost standardized tooth preparation.

In the present study, patients recall was performed after 6 months, 12 months, 18 months, 24 months and 30 months for clinical evaluation according to the clinical evaluation criteria (modified USPHS criteria for indirect restorations). It was found that At 6 months, score A was recorded in all specimens with no statistically significant difference between different surface treatments $(\mathrm{p}=1)$. At 12 months, score A was recorded in all specimens with no statistically significant difference between different surface treatments $(\mathrm{p}=1)$. At 18 months, score A was recorded in $100 \%$ specimens of CoJet and acid mixture surface treatment, in $90 \%$ of specimens of no treatment and in $80 \%$ of specimens of sandblasting and SIE; with no statistically significant difference between different surface treatments $(\mathrm{p}=0.815)$. At 24 months, score A was recorded in $80 \%$ specimens of sandblasting, CoJet and acid mixture surface treatment, in $66.7 \%$ of specimens of no treatment and in $22.2 \%$ of SIE; with a statistically significant difference between different surface treatments $(\mathrm{p}=0.045)$. At 30 months, score A was recorded in $80 \%$ specimens of sandblasting, $88.9 \%$ CoJet and $77.8 \%$ of acid mixture surface treatment, in $44.5 \%$ of specimens of no treatment and in $11.1 \%$ of SIE; with a statistically significant difference between different surface treatments $(\mathrm{p}=0.0024)$. Failure was detected in some clinical cases within different groups (figure 4). This failure was assessed according to modified Ryge criteria as Bravo; Dull surface and/or chipping of porcelain that does not impair function. This failure mode starts to appear at 18 months in some groups. Other cases were reported as Charlie; Chipping of the veneering porcelain impairing esthetics and exposing framework material. However, this type of failure mode appears at 24 months recall period 
in few cases receiving SIE surface treatment and 30 months recall period in few cases in SIE group and no treatment group. These observations might be attributed to the difference in the coefficient of thermal expansion of the molten infiltrating glass present at the newly created interface after SIE treatment and the veneering ceramic materials. Furthermore, by looking deeply in the results of the present study it could be addressed that the treatment of the surface zirconia framework with Cojet system was able to maintain acceptable clinical performance at 24 months and 30 months recall periods. However, the other surface treatments showed lesser performance. This might be attributed to that treatment with Cojet system provide a double benefits to the surface of zirconia framework both mechanical represented by surface roughness as well as chemical by silicatization. However, other surface treatments that create only surface roughness might induce a kind of internal stresses and consequently microflaws that may accelerate the "pop-off" of overlying ceramic. Regarding the surface treatment with the novel acid mixture, the results reveals that the clinical performance was excellent in 6,12 and 18 months recall periods that start to decrease to bravo scoring (clinically acceptable with minimal defect not impairing function) in 24 and 30 months recall periods, however no Charlie failure mode was detected in the current study. This was in accordance with previous studies ${ }^{(13,14)}$ who stated that the acid mixture might have increased the contact area between the zirconia core and veneering ceramic that might be responsible for the good bond strength between zirconia framework and veneering porcelain. It was also reported that the failure mode detected in the previous studies was mainly cohesive with veneering porcelain where a thin porcelain layer still remained on the zirconia cores. This type of failure indicates the good interfacial bond between the core and the veneer material. So it could be assumed that under clinical conditions where fatigue is the dominant factor contributing to failure, bond reduction is even more expected and could lead to delamination of veneering ceramic. Under such conditions, selection of the bonding mechanism should focus equally on long-term bond strength ability as well as the initial high bond strength value.

This result was in agreement with Rosentritt et al 2009 ${ }^{(17)}$ who stated that the failure mode showed that the failure depends on the strength of the weakest part of the crown which seems to be the veneering ceramic and to a lesser extent on the bond at the core-veneer interface but not on the strength of the underlying core structures. In all cases, the tested systems showed chipping of the comparable low-strength veneering ceramic but no fracture of the high-strength core or the natural tooth in clinical cases.

The chipping of the veneering porcelain at functional cusp observed in the current study was also in accordance with Çömlekoğlu et al $\mathbf{2 0 0 8}^{(18)}$ who stated that in all ceramic restorations, compressive and tensile stresses have been reported to accumulate on heavy load bearing areas. The veneering ceramics should therefore have high bond strengths to their frameworks in order to resist these stresses and thereby prevent chipping of the restoration at this region.

The result of the present study was also consistent with Liu et al $\mathbf{2 0 1 0}^{(19)}$ who stated that by bearing mind the fact that interfacial delamination starts well before the crack propagates through the Y-TZP layer, one may understand that under the fatigue loading conditions local veneer flaking may occur even though no critical damage of the Y-TZP core itself has taken place. This could be explained as in case of Y-TZP bilayer composite, the flexural strength of Y-TZP is ten times higher than that of the veneer porcelain which is high enough to initiate the process of dynamic breaking of the veneer layer.

Another issue that should be addressed regarding the failure in the present study was that mentioned by Fischer et al $2008^{(20)}$ who stated that the fact that 
the strength of veneering ceramics for zirconia is in the same order as that of veneering materials for metal-ceramics and could be interpreted in the sense that the strength of the veneering ceramics are not the limiting factor for the clinical long-term success of zirconia restorations. Nevertheless, compared to metal-ceramics excessive chipping is observed in clinical studies with zirconia restorations. To explain this effect, two aspects have to be considered. One aspect is the stress built during cooling after firing of the veneering ceramic. In metal-ceramic systems, this stress may be at least partially relaxed by an elastic or plastic deformation of the substructure especially high-gold alloys which show a low sagresistance. A zirconia substructure in contrast is rigid which leads to higher stress formation. Hence, compared to metal-ceramics a higher flexural strength of the veneering ceramic is favorable to provide a high reliability of the veneer. Therefore, the effort to improve the veneering ceramics for zirconia should be directed to the optimal adjustment of the thermal expansion and the increase of mechanical strength. A second point is the fact that in the oral cavity water exposure may cause hydrolysis of the $\mathrm{Si}-\mathrm{O}-\mathrm{Si}$ bonds, thus affecting the mechanical properties of the ceramic.

Another rational for evaluation of longterm clinical performance of zirconia supported restoration was introduced by White et al $\mathbf{2 0 0 5}^{(21)}$ who concluded that the poor interfacial bond strength is firstly due to superior ability of zirconia core to resist tensile stresses created within the specimens, secondly it may be due to relatively poor or less than perfect bond between zirconia core and veneering ceramic which is the consequence of either residual stresses remained from zirconia sintering, porcelain firing or surface treatment procedures. The clinical implication of this finding is that this system could have a tendency to produce porcelain "pop-off" rather than catastrophic failure. Of course, any type of damage is unwelcome, but "pop-off" might be considered a lesser evil.

Finally, it could be addressed that flaking of veneering ceramic in zirconia based restoration is still a debatable issue which is depending on several factors such as lack of proper framework support, internal defects, mismatch between the thermal coefficients $(t)$ of the veneering and core materials, direction, magnitude and frequency of the applied load as well as the residual stresses induced by processing. All these factors were reported to be responsible for the cause of fracture of veneering ceramic on ceramic core materials. Compressive stresses are generated in the veneering ceramic as a result of differences in $(t)$ of both the framework and the veneering ceramics and this is in accordance with Çömlekoğlu et al 2008 ${ }^{(18)}$.

\section{CONCLUSIONS}

Under the limitations of this study the following conclusions could be derived:

1) In all cases, the tested systems showed chipping of the comparable low-strength veneering ceramic but no fracture of the high-strength core or the natural tooth in clinical cases.

2) The treatment of the surface zirconia framework with Cojet system was able to maintain acceptable clinical performance at 24 months and 30 months recall periods. However, the other surface treatments showed lesser performance.

3) No direct correlation between zirconia framework surface treatments and long term bonding to veneering ceramic could be addressed in the current study; however, it could be multifactorial such as lack of proper framework support, internal defects, direction, magnitude and frequency of the applied load as well as the residual stresses induced by processing.

\section{RECOMMENDATIONS}

Further controlled clinical trials may be required to reveal the secrets behind the impact of surface treatments of zirconia framework on the long term clinical performance all ceramic zirconia-veneered crowns. 


\section{REFERENCES}

1. Raigrodski AJ.: Contemporary materials and technologies for all-ceramic fixed partial dentures: a review of the literature. J Prosthet Dent 2004;92:557-62.

2. Sundh A, Molin M and Sjogren G.: Fracture resistance of yttrium oxide partially-stabilized zirconia all-ceramic bridges after veneering and mechanical fatigue testing. Dent Mater 2005;21:476-482.

3. Aboushelib MN, de Jager N, Kleverlaan CJ and Feilzer AJ.: Effect of loading method on the fracture mechanics of two layered all-ceramic restorative systems. Dent Mater 2007;23:952-9.

4. Fleming GJP, Dickens M, Thomas LJ and Harris JJ.: The in vitro failure of all-ceramic crowns and the connector area of fixed partial dentures using bilayered ceramic specimens: The influence of core to dentine thickness ratio. Dent Mater 2006;22:1-7.

5. Vult Von Steyern P, Carlson P and Nilner K.: All-ceramic fixed partial dentures designed according to the DCZircon technique. A 2-year clinical study. J Oral Rehabil 2005;32:180-7.

6. Sailer I, Fehér A, Filser F, Lüthy H, Gauckler LJ, Scharer $\mathrm{P}$ and Hammerle CHF.: Prospective clinical study of zirconia posterior fixed partial dentures: 3-year follow-up. Quintessence Int 2006;37:41-9.

7. Sailer I, Fehér A, Filser F, Gauckler LJ, Lüthy H and Hammerle CHF.: Five-year clinical results of zirconia frameworks for posterior fixed partial dentures. Int J Prosthodont 2007;20:383-8.

8. Pjetursson BE, Sailer I, Zwahlen M and Hammerle CHF.: A systematic review of the survival and complication rates of all-ceramic and metal-ceramic reconstructions after an observation period of at least 3 years. Part I: single crowns. Clin Oral Impl Res 2007;18:73-85.

9. De Jager N, Pallav $\mathrm{P}$ and Feilzer AJ.: The influence of design parameters on the FEA-determined stress distribution in CAD-CAM produced all-ceramic dental crowns. Dent Mater 2005;21:242-251.

10. Saito A., Komine F., Blatz M. B., and Matsumura H.: A comparison of bond strength of layered veneering porcelains to zirconia and metal. J Prosthet Dent 2010;104:247-257
11. Aboushelib MN and Wang H.J.: Effect of surface treatment on flexural strength of zirconia bars. Prosthet Dent. 2010; 104(2):98-104.

12. Haitham A. Mohamed; Jihan F. Younis and Tarek S. Morsi: Microtensile bond strength of veneering ceramic to cadcam zirconia after different surface treatments. E.D.J. 2013 Vol. 59, No. 2

13. Amr S. El-Etreby and Marwa M. Wahsh: Bond strength of veneering porcelain and resin cement to zirconia core treated with a novel acid mixture.E.D.J. 2015 Vol. 61,No. 3

14. Marwa M. Wahsh and Amr S. El-Etreby: Effect of a novel surface treatment on the biaxial flexural strength of zirconia ceramic. E.D.J. 2015 Vol. 61, No. 2

15. Etman MK and Woolford MJ.: Three-year clinical evaluation of two ceramic crown systems: a preliminary study. J Prosthet Dent. 2010;103(2):80-90.

16. Casucci A, Mazzitelli C, Monticelli F, Toledano M, Osorio R, Osorio E, Papacchini F and Ferrari M.: Morphological analysis of three zirconium oxide ceramics: Effect of surface treatments. Dent Mater. 2010;26(8):751-60. Epub 2010 May 14.

17. Rosentritt $M$, Behr $M$, Thaller C, Rudolph $H$ and Feilzer A.: Fracture performance of computer-aided manufactured zirconia and alloy crowns. Quintessence Int. 2009;40(8):655-62.

18. Çömlekoğlu M. E, Dündar M, Özcan M, Güngör M. A, Gökçe B and Artunç C.: Evaluation of bond strength of various margin ceramics to a zirconia ceramic. Journal of dentistry 2008;36:822 - 827

19. Liu Y., Feng H., Bao Y., Qiu Y., Xing N. and Shen $\mathrm{Z}$.: Fracture and interfacial delamination origins of bilayer ceramic composites for dental restorations. Journal of the European Ceramic Society 2010; 30:1297-1305.

20. Fischer J., Stawarczyk B. and Hämmerle C.H.F.: Flexural strength of veneering ceramics for zirconia. journal of dentistry 2008;36:316 - 321

21. White S.N., Miklus V.G., E.A. McLaren, Lang L.A. and Caputo A.A.: Flexural strength of a layered zirconia and porcelain dental all-ceramic system.J Prosthet Dent 2005;94:125-31. 